\title{
Completing partial Latin squares with two filled rows and two filled columns
}

\author{
Peter Adams, Darryn Bryant and Melinda Buchanan \\ pa@maths.uq.edu.au, db@maths.uq.edu.au, m.buchanan@uq.edu.au \\ Department of Mathematics, University of Queensland, QLD 4072, Australia \\ Submitted: Jul 16, 2007; Accepted: Apr 8, 2008; Published: Apr 18, 2008 \\ Mathematics Subject Classification: 05B15
}

\begin{abstract}
It is shown that any partial Latin square of order at least six which consists of two filled rows and two filled columns can be completed.
\end{abstract}

\section{Introduction}

Problems concerning the completion of partial Latin squares are notoriously difficult. It is known that deciding whether an arbitrary partial Latin square has a completion is NPcomplete [4]. Several classical results in the field concern completability for large families of partial Latin squares. Historically, one of the most significant of these is encapsulated by Evans' Conjecture, posed in 1960 by Trevor Evans [5]. Smetaniuk published a proof of the conjecture in 1981 [12].

Theorem 1.1 [12] Any partial Latin square of order $n$ with at most $n-1$ filled cells is completable.

Smetaniuk's result completed Lindner's partial proof of 1970 [9]. Several other partial results were published, notably by Marica and Schönheim [10] and Häggkvist [6].

In 1983, Andersen and Hilton [1] published an alternative proof. Their paper includes the stronger result, stated in Theorem 1.2, of the necessary and sufficient conditions for a partial Latin square of order $n$ to be completed if at most $n$ cells are filled.

Theorem 1.2 [1] A partial Latin square of order $n$ with at most $n$ filled cells is completable if and only if it is not isotopic to one of the partial Latin squares in the figure below. 

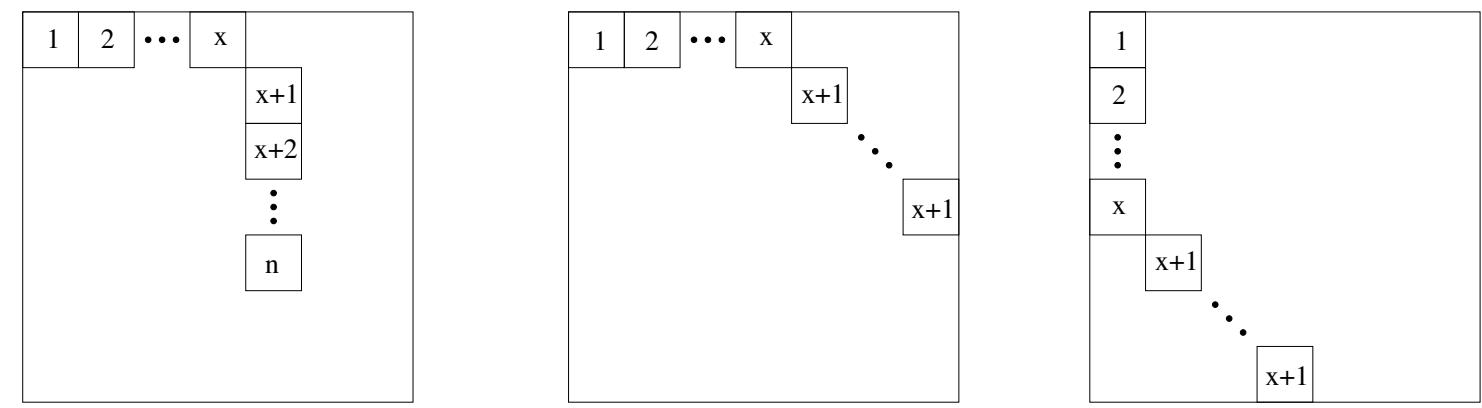

In 1945, M. Hall [7] established the following result using P. Hall's Theorem [8] on the existence of systems of distinct representatives of subsets.

Theorem 1.3 [7] Any partial Latin square of order $n$ consisting of $r$ filled rows, $r \leq n$, can be completed.

Theorem 1.4 below is a famous result of Ryser from 1951. It extends Theorem 1.3.

Theorem 1.4 [11] A partial Latin square of order $n$ in which cell $(i, j)$ is filled precisely when $1 \leq i \leq r$ and $1 \leq j \leq s$ for some $r, s \leq n$ can be completed if and only if each symbol occurs at least $r+s-n$ times in the filled cells.

In Theorem 1.4, the filled cells form an $r$ by $s$ rectangular array. It is natural then to ask about completions of partial Latin squares in which the empty cells form a rectangular array; that is, partial Latin squares in which certain rows and columns are filled and all other cells are empty. If the number of filled rows or the number of filled columns is one, then completability follows as an easy consequence of Theorem 1.3. In this paper we prove the following theorem which gives a complete solution in the case of two filled rows and two filled columns.

Theorem 1.5 Let $P$ be a partial Latin square with two filled rows, two filled columns and all other cells empty. Then $P$ is completable if and only if $P$ is not isotopic to either of the following two partial Latin squares.

\begin{tabular}{|l|l|l|l|}
\hline 1 & 2 & 3 & 4 \\
\hline 2 & 3 & & \\
\hline 3 & 4 & 2 & 1 \\
\hline 4 & 1 & & \\
\hline
\end{tabular}

\begin{tabular}{|l|l|l|l|l|}
\hline 1 & 2 & 3 & 4 & 5 \\
\hline 2 & 3 & & & \\
\hline 3 & 1 & 2 & 5 & 4 \\
\hline 4 & 5 & & & \\
\hline 5 & 4 & & & \\
\hline
\end{tabular}

A complete proof of this theorem is given in Buchanan's $\mathrm{PhD}$ thesis [3] and is over 100 pages long. The proof splits into numerous cases and involves lengthy but routine arguments which are somewhat repetitive in places. Here, by referring the reader to [3] for many such arguments, we are able to give a condensed version of the proof which covers the main ideas and constructions. The proof is essentially along the same lines as that of the corresponding result for symmetric Latin squares which was proven in [2]. However, 
as we shall see in later sections, the techniques from [2] need to be considerably extended for the problem under consideration here.

We give necessary basic definitions and concepts in Section 2 and state several preliminary results in Section 3. In Section 4 we define a class of partial Latin squares, see Definition 4.2, which is central to the proof of the main result. Conditions (4.1) - (4.5) and (5.1) - (5.3) of this definition determine the division into cases of the main construction, which is given in Section 6. Section 5 gives a basic outline of the main construction and also establishes further notation which will be used in Section 6 .

\section{Definitions and Notation}

In this section we introduce some terminology and concepts which will be used throughout this paper.

A partial Latin square $S$ of order $n$ is a set of ordered triples of the form $(i, j, k)$ with $i, j$ and $k$ each chosen from an $n$-set such that distinct triples never agree in more than one coordinate. A (complete) Latin square of order $n$ is a partial Latin square of order $n$ with $n^{2}$ triples. The underlying set is usually $\{1,2, \ldots, n\}$ and it is usual to write a partial Latin square $S$ as an $n$ by $n$ array of cells with symbol $k$ in row $i$ and column $j$, that is, in cell $(i, j)$, if and only if $(i, j, k) \in S$. Two partial Latin squares $P_{1}$ and $P_{2}$ are isotopic if $P_{2}$ can be obtained from $P_{1}$ by applying a sequence of row, column or symbol permutations.

A cell of a partial Latin square is filled if it contains a symbol and empty otherwise. A row or column is filled if all of its cells are filled, empty if all of its cells are empty, and partially filled if it is neither filled nor empty. Given a partial Latin square $P$, we say that symbol $k$ is allowable for cell $(i, j)$, and that $(i, j)$ is an allowable cell for symbol $k$, if $P \cup\{(i, j, k)\}$ is a partial Latin square.

A partial Latin square $P$ of order $n$ is completable if there exists a Latin square $L$ of order $n$ with $P \subseteq L$, and $L$ is called a completion of $P$. A row $i$ (column $j$ ) in a partial Latin square $P$ is completable if there exists a partial Latin square $P^{\prime}$, of the same order as $P$, such that $P \subseteq P^{\prime}$ and row $i$ (column $j$ ) of $P^{\prime}$ is filled.

A set $S$ is a subsquare of a partial Latin square $P$ if $S \subseteq P$ and $S$ is a Latin square. Suppose a Latin square contains the triples $\left(i_{1}, j_{1}, x\right),\left(i_{2}, j_{2}, x\right),\left(i_{1}, j_{2}, y\right)$ and $\left(i_{2}, j_{1}, y\right)$. We shall call such a configuration of cells and symbols an $\left(i_{1}, j_{1}, i_{2}, j_{2}, x, y\right)$-intercalate. If we replace these triples with $\left(i_{1}, j_{1}, y\right),\left(i_{2}, j_{2}, y\right),\left(i_{1}, j_{2}, x\right)$ and $\left(i_{2}, j_{1}, x\right)$, we obtain a new Latin square which we say is obtained from the original by switching in the intercalate.

In a partial Latin square $P$, we say two symbols $x$ and $y$ in cells $\left(i_{1}, j_{1}\right)$ and $\left(i_{2}, j_{2}\right)$ respectively, are swappable if replacing the triples $\left(i_{1}, j_{1}, x\right)$ and $\left(i_{2}, j_{2}, y\right)$ of $P$ with triples $\left(i_{1}, j_{1}, y\right)$ and $\left(i_{2}, j_{2}, x\right)$ results in a partial Latin square.

Throughout the paper the Latin squares of main interest will be of order $n$. We will denote $\left\lfloor\frac{n}{2}\right\rfloor$ by $m$ and we will often need to distinguish between the integers $1,2, \ldots, m$ and the integers $m+1, m+2, \ldots, n$. Thus we introduce the following notation. An integer or symbol $i$ will be called small if $1 \leq i \leq m$ and large if $m+1 \leq i \leq n$. Column $j$ or cell $(i, j)$ is in the left of a partial Latin square if $j$ is small and is in the right if $j$ is large. 
Similarly, row $i$ or cell $(i, j)$ is in the top of a partial Latin square if $i$ is small and is in the bottom if $i$ is large. Our main construction involves partitioning Latin squares into four parts; the top left, top right, bottom left and bottom right.

We define a row $i$ of a partial (or complete) Latin square of order $n$ to be segregated if it satisfies one of the following statements.

- Row $i$ is in the top, all symbols in the left of row $i$ are small and all symbols in the right of row $i$ are large.

- Row $i$ is in the bottom, $n$ is even, all symbols in the left of row $i$ are large and all symbols in the right of row $i$ are small.

- Row $i$ is in the bottom, $n$ is odd, all symbols in the left of row $i$ are large and the right of row $i$ contains at most one large symbol.

Similarly, we define a column $j$ of a partial (or complete) Latin square of order $n$ to be segregated if it satisfies one of the following statements.

- Column $j$ is in the left, all symbols in the top of column $j$ are small and all symbols in the bottom of column $j$ are large.

- Column $j$ is in the right, $n$ is even, all symbols in the top of column $j$ are large and all symbols in the bottom of column $j$ are small.

- Column $j$ is in the right, $n$ is odd, all symbols in the top of column $j$ are large and the bottom of column $j$ contains at most one large symbol.

A segregated partial Latin square of order $n$ is a partial Latin square of order $n$ such that each row and each column is segregated, and such that if $n$ is odd then the bottom right contains at most $m+1$ large symbols, all of which are distinct and no two of which occur in the same row or in the same column.

The additional condition in the case $n$ is odd is included because we want a partial Latin square such as

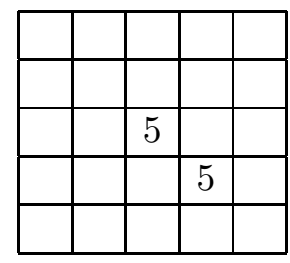

to be not segregated, even though all its rows and columns are segregated. It is easy to see that a partial Latin square of odd order which does not satisfy this additional property cannot be completed to a segregated Latin square. For example, the partial Latin square above cannot be completed to a segregated Latin square, as any completion necessarily contains an occurrence of the symbol 5 in the top left. Segregated partial Latin squares play an important role in the constructions in later sections. 
Let $L$ be a segregated Latin square of odd order and let $s$ be a large symbol. For example, see the segregated Latin square of order 9 below. There is precisely one column in the top right which does not contain $s$, and there is precisely one row in the bottom left which does not contain $s$. Thus, the bottom right of $L$ contains precisely one occurrence of each of the $m+1$ large symbols. Furthermore, no two of these large symbols occur in the same row or in the same column in the bottom right. The remaining cells in the bottom right of $L$ are filled with small symbols.

When $n$ is odd it is sometimes convenient to append some additional cells as follows to help describe our constructions. For each large symbol $s$ we append two additional cells $(r, n+1)$ and $(n+1, c)$, each containing $s$, where $(r, c)$ is the unique cell in the bottom right containing $s$. Then cells $(i, j)$ with $i \in\{1,2, \ldots, m\} \cup\{n+1\}$ and $j \in\{m+1, m+2, \ldots, n\}$ form a Latin square which we call the augmented top right. Similarly, cells $(i, j)$ with $i \in\{m+1, m+2, \ldots, n\}$ and $j \in\{1,2, \ldots, m\} \cup\{n+1\}$ form a Latin square which we call the augmented bottom left. The augmented left of some row, say $r$, in the bottom of a partial Latin square of odd order is the left of row $r$ with the cell $(r, n+1)$ appended to it.

\begin{tabular}{|c|c|c|c|c|c|c|c|c|}
\hline 4 & 1 & 2 & 3 & 9 & 5 & 7 & 6 & 8 \\
\hline 3 & 4 & 1 & 2 & 8 & 7 & 9 & 5 & 6 \\
\hline 1 & 2 & 3 & 4 & 5 & 6 & 8 & 7 & 9 \\
\hline 2 & 3 & 4 & 1 & 6 & 9 & 5 & 8 & 7 \\
\hline 7 & 9 & 5 & 8 & 1 & 4 & 6 & 2 & 3 \\
\hline 5 & 7 & 9 & 6 & 3 & 8 & 1 & 4 & 2 \\
\hline 6 & 5 & 8 & 7 & 4 & 3 & 2 & 9 & 1 \\
\hline 9 & 8 & 6 & 5 & 7 & 2 & 3 & 1 & 4 \\
\hline 8 & 6 & 7 & 9 & 2 & 1 & 4 & 3 & 5 \\
\hline
\end{tabular}

Henceforth, wherever possible we shall adopt a convention of labeling rows with letters from the Greek alphabet, labeling columns with upper case letters from the English alphabet and labeling symbols with lower case letters from the English alphabet.

\section{Preliminary Results}

The lemmas in this section will be used in Sections 5 and 6. Since a partial Latin square $P$ is completable if and only if its transpose is completable, the lemmas in this section also hold when the words "row" and "column" are interchanged. Lemmas 3.1 and 3.2 are easy corollaries of Theorem 1.3, and Lemma 3.3 follows easily from P. Hall's Theorem [8] (see [3] for proofs). The proofs of Lemmas 3.4-3.8 involve routine applications of results from [6] and [8], and are omitted here. The proofs can be found in [3].

Lemma 3.1 Let $P$ be a partial Latin square of order $n$ with $x$ filled columns, with one filled row $\gamma$ and with all other cells empty. Then $P$ is completable. 
Lemma 3.2 Let $P$ be a completable partial Latin square of order $n$ and let $s \in\{1,2, \ldots, n\}$. Let $Q$ be any partial Latin square of order $n$ such that $P \subseteq Q$ and such that if $(\gamma, C, t) \in Q \backslash P$ then $t=s$ and row $\gamma$ is empty in $P$. Then $Q$ is completable.

Lemma 3.3 Let $G$ be a spanning subgraph of the complete bipartite graph $K_{x, x}$ with minimum degree at least $\frac{x}{2}$. Then $G$ contains a perfect matching.

Lemma 3.4 Let $x \leq n$ and let $P$ be a partial Latin square of order $n$ with $x$ filled columns, with one filled row $\alpha$, with one partially filled row $\beta$ containing at least two empty cells, and with all other cells empty. Then row $\beta$ is completable.

Lemma 3.5 Let $x \leq n$ and let $P$ be a partial Latin square of order $n$ with $x$ filled columns, with one filled row $\alpha$, with one partially filled row $\beta$ having at least three empty cells, and with exactly one other filled cell. Then row $\beta$ is completable.

Lemma 3.6 Let $P$ be a partial Latin square of order $n \geq 8$ with two filled rows $\alpha$ and $\beta$, and one partially filled row $\gamma$ with at most three filled cells. Suppose further that at most two cells in rows other than $\alpha, \beta$ and $\gamma$ are filled, and that these filled cells contain distinct symbols and occur in distinct columns. Then row $\gamma$ is completable.

Lemma 3.7 Let $P$ be a partial Latin square of order $n \geq 8$ with three filled rows $\alpha, \beta$ and $\gamma$ and at most three other filled cells. Then $P$ is completable unless

(1) $n=8$;

(2) row $\epsilon$ of $P$ is partially filled and contains precisely three filled cells $\left(\epsilon, C_{1}, s_{1}\right)$, $\left(\epsilon, C_{2}, s_{2}\right)$ and $\left(\epsilon, C_{3}, s_{3}\right)$; and

(3) $P$ contains a subsquare of order 3 in rows $\alpha, \beta$ and $\gamma$ and in columns other than $C_{1}, C_{2}$ and $C_{3}$, based on symbols from the set $\{1,2, \ldots, n\} \backslash\left\{s_{1}, s_{2}, s_{3}\right\}$.

Lemma 3.8 Let $P$ be a partial Latin square of order $n \geq 7$ with one or two filled rows and at most four other filled cells. If each partially filled row of $P$ contains at most three filled cells, then $P$ is completable.

\section{Reduction of the Problem}

In this section we show that we only need to consider partial Latin squares of a particular form. The following observation is crucial and its proof is an easy exercise.

Lemma 4.1 If a partial Latin square $P$ is isotopic to $P^{\prime}$, then $P$ is completable if and only if $P^{\prime}$ is completable. 
We shall need some further definitions. Let $\mathcal{Q}_{n}$ denote the class of partial Latin squares of order $n$ having two filled rows, two filled columns, and with all other cells empty. For any partial Latin square $Q \in \mathcal{Q}_{n}$ we define $\alpha=\alpha(Q)$ and $\beta=\beta(Q)$ by $\alpha, \beta \in\{1,2, \ldots, n\}$ and row $\alpha$ and row $\beta$ are the filled rows of $Q$ with $\alpha<\beta$.

Let columns $C_{1}=C_{1}(Q)$ and $C_{2}=C_{2}(Q)$ be the filled columns of $Q \in \mathcal{Q}_{n}$ and let $\Lambda=\Lambda(Q)$ be the permutation of $\{1,2, \ldots, n\}$ defined by $\Lambda(x)=y$ if and only if $x$ and $y$ are the symbols in columns $C_{1}$ and $C_{2}$ respectively of some row of $Q$. Let $\omega$ be the number of cycles in $\Lambda$ when it is expressed as a product of disjoint cycles. Note that $\Lambda$ has no fixed points. The column cycle type of $Q$ is the multiset whose $\omega$ elements are the lengths of the cycles in $\Lambda$. If a cycle in $\Lambda$ contains exactly one of the symbols in cells $\left(\alpha, C_{1}\right)$ and $\left(\beta, C_{1}\right)$ then we shall refer to it as starred and we append ${ }^{*}$ to its length in the column cycle type. If a single cycle contains both the symbols in cells $\left(\alpha, C_{1}\right)$ and $\left(\beta, C_{1}\right)$ then we shall refer to it as double starred and we append ${ }^{* *}$ to its length in the column cycle type.

The partial Latin square shown on the left below has column cycle type $\left\{2^{*}, 2,3^{*}\right\}$ and its transpose shown on the right has column cycle type $\left\{5^{* *}, 2\right\}$.

\begin{tabular}{|l|l|l|l|l|l|l|}
\hline & & 5 & & 7 & & \\
\hline & & 1 & & 3 & & \\
\hline & & 2 & & 4 & & \\
\hline 6 & 3 & 7 & 1 & 5 & 4 & 2 \\
\hline & & 3 & & 1 & & \\
\hline 5 & 1 & 6 & 3 & 2 & 7 & 4 \\
\hline & & 4 & & 6 & & \\
\hline
\end{tabular}

\begin{tabular}{|l|l|l|l|l|l|l|}
\hline & & & 6 & & 5 & \\
\hline & & & 3 & & 1 & \\
\hline 5 & 1 & 2 & 7 & 3 & 6 & 4 \\
\hline & & & 1 & & 3 & \\
\hline 7 & 3 & 4 & 5 & 1 & 2 & 6 \\
\hline & & & 4 & & 7 & \\
\hline & & & 2 & & 4 & \\
\hline
\end{tabular}

We shall see soon that for any partial Latin square $Q \in \mathcal{Q}_{n}$ of order $n \geq 14$, either $Q$ or $Q^{T}$ is isotopic to a partial Latin square $P \in \mathcal{P}_{n}$, where $\mathcal{P}_{n}$ is the subclass of $\mathcal{Q}_{n}$ which we define in Definition 4.2 below. For example, consider the partial Latin squares $Q, Q^{\prime}, Q^{\prime \prime}$ and $P$ shown below. The partial Latin square $Q \in \mathcal{Q}_{14}$ and is isotopic to $P \in \mathcal{P}_{14}$.

\begin{tabular}{|l|l|l|l|l|l|l|l|l|l|l|l|l|l|}
\hline & & & & & 12 & & & & 10 & & & & \\
\hline & & & & & 6 & & & & 2 & & & & \\
\hline & & & & & 3 & & & & 8 & & & & \\
\hline & & & & & 14 & & & & 9 & & & & \\
\hline & & & & & 13 & & & & 5 & & & & \\
\hline & & & & & 10 & & & & 1 & & & & \\
\hline & & & & & 2 & & & & 3 & & & & \\
\hline & & & & & 11 & & & & 13 & & & & \\
\hline 2 & 10 & 14 & 13 & 12 & 1 & 11 & 3 & 7 & 4 & 8 & 9 & 6 & 5 \\
\hline & & & & & 8 & & & & 7 & & & & \\
\hline 13 & 14 & 7 & 11 & 10 & 9 & 5 & 1 & 8 & 6 & 12 & 3 & 4 & 2 \\
\hline & & & & & 4 & & & & 12 & & & & \\
\hline & & & & & 7 & & & & 14 & & & & \\
\hline & & & & & 5 & & & & 11 & & & & \\
\hline
\end{tabular}


If we first apply the permutation

$$
(1,4,11,5,14,12,3,8,13,10,9,2,6)(7)
$$

to the rows of $Q$, then we obtain the partial Latin square $Q^{\prime}$.

\begin{tabular}{|c|c|c|c|c|c|c|c|c|c|c|c|c|c|}
\hline & & & & & 10 & & & & 1 & & & & \\
\hline 2 & 10 & 14 & 13 & 12 & 1 & 11 & 3 & 7 & 4 & 8 & 9 & 6 & 5 \\
\hline & & & & & 4 & & & & 12 & & & & \\
\hline & & & & & 12 & & & & 10 & & & & \\
\hline 13 & 14 & 7 & 11 & 10 & 9 & 5 & 1 & 8 & 6 & 12 & 3 & 4 & 2 \\
\hline & & & & & 6 & & & & 2 & & & & \\
\hline & & & & & 2 & & & & 3 & & & & \\
\hline & & & & & 3 & & & & 8 & & & & \\
\hline & & & & & 8 & & & & 7 & & & & \\
\hline & & & & & 7 & & & & 14 & & & & \\
\hline & & & & & 14 & & & & 9 & & & & \\
\hline & & & & & 5 & & & & 11 & & & & \\
\hline & & & & & 11 & & & & 13 & & & & \\
\hline & & & & & 13 & & & & 5 & & & & \\
\hline
\end{tabular}

Applying the permutation

$$
(1,2,7,10)(3,8,9,5,12,4)(11,13,14)(6)
$$

to the symbols of $Q^{\prime}$ gives the partial Latin square $Q^{\prime \prime}$.

\begin{tabular}{|l|l|l|l|l|l|l|l|l|l|l|l|l|l|}
\hline & & & & & 1 & & & & 2 & & & & \\
\hline 7 & 1 & 11 & 14 & 4 & 2 & 13 & 8 & 10 & 3 & 9 & 5 & 6 & 12 \\
\hline & & & & & 3 & & & & 4 & & & & \\
\hline & & & & & 4 & & & & 1 & & & & \\
\hline 14 & 11 & 10 & 13 & 1 & 5 & 12 & 2 & 9 & 6 & 4 & 8 & 3 & 7 \\
\hline & & & & & 6 & & & & 7 & & & & \\
\hline & & & & & 7 & & & & 8 & & & & \\
\hline & & & & & 8 & & & & 9 & & & & \\
\hline & & & & & 9 & & & & 10 & & & & \\
\hline & & & & & 10 & & & & 11 & & & & \\
\hline & & & & & 11 & & & & 5 & & & & \\
\hline & & & & & 12 & & & & 13 & & & & \\
\hline & & & & & 13 & & & & 14 & & & & \\
\hline & & & & & 14 & & & & 12 & & & & \\
\hline
\end{tabular}

We obtain the partial Latin square $P$ by applying the permutation

$$
(1,7,8,10,2,3,14,11,12,5,4,9,13,6)
$$

to the columns of $Q^{\prime \prime}$. It may be useful to refer to $P$ when reading Definition 4.2. 


\begin{tabular}{|c|c|c|c|c|c|c|c|c|c|c|c|c|c|}
\hline 1 & 2 & & & & & & & & & & & & \\
\hline 2 & 3 & 1 & 4 & 5 & 6 & 7 & 13 & 14 & 8 & 12 & 9 & 10 & 11 \\
\hline 3 & 4 & & & & & & & & & & & & \\
\hline 4 & 1 & & & & & & & & & & & & \\
\hline 5 & 6 & 11 & 1 & 8 & 3 & 14 & 12 & 13 & 2 & 7 & 4 & 9 & 10 \\
\hline 6 & 7 & & & & & & & & & & & & \\
\hline 7 & 8 & & & & & & & & & & & & \\
\hline 8 & 9 & & & & & & & & & & & & \\
\hline 9 & 10 & & & & & & & & & & & & \\
\hline 10 & 11 & & & & & & & & & & & & \\
\hline 11 & 5 & & & & & & & & & & & & \\
\hline 12 & 13 & & & & & & & & & & & & \\
\hline 13 & 14 & & & & & & & & & & & & \\
\hline 14 & 12 & & & & & & & & & & & & \\
\hline
\end{tabular}

Definition 4.2 Let $n \geq 14$. A partial Latin square $P$ of order $n$ is in the class $\mathcal{P}_{n}$ if and only if $P \in \mathcal{Q}_{n}$ and $P$ satisfies Conditions (1)-(15) below, where $m_{1}, m_{2}, \ldots, m_{\omega}$ is a list of the elements of the column cycle type of $P$ and where $\sigma_{0}=0$ and $\sigma_{i}=m_{1}+m_{2}+\ldots+m_{i}$ for $i=1,2, \ldots, \omega$.

(1) Columns 1 and 2 of $P$ are the filled columns.

(2) Column 1 is $(1,2, \ldots, n)$.

(3) Column 2 of $P$ is $(\pi(1), \pi(2), \ldots, \pi(n))$ where for $i=1,2, \ldots, n, \pi(i)=i+1$ unless $i \in\left\{\sigma_{1}, \sigma_{2}, \ldots, \sigma_{\omega}\right\}$ in which case $\pi\left(\sigma_{j}\right)=\sigma_{j-1}+1$.

(4) If $n$ is even then for some $r \in\{1,2, \ldots, \omega\}$, at least one of the following statements holds:

(4.1) $\sigma_{r}=m$.

(4.2) $\sigma_{r-1} \leq m-2$ and $\sigma_{r} \geq m+2$.

(4.3) $\sigma_{r-1} \leq m-2$ and $\sigma_{r}=m+1$.

(4.4) $\sigma_{r-1}=m-1$ and $\sigma_{r} \geq m+2$.

(4.5) $\sigma_{r-1}=m-1$ and $\sigma_{r}=m+1$.

(5) If $n$ is odd then for some $r \in\{1,2, \ldots, \omega\}$, at least one of the following statements holds:

(5.1) $\sigma_{r}=m$.

(5.2) $\sigma_{r-1} \leq m-2$ and $\sigma_{r} \geq m+2$.

(5.3) $\sigma_{r-1}=m-1$ and $\sigma_{r} \geq m+2$. 
(6) If $P$ satisfies Condition (4.5) then $m$ is odd, $P$ has column cycle type $\left\{2^{*}, 2^{*}, 2,2, \ldots, 2\right\},\left\{2^{* *}, 2,2, \ldots, 2\right\}$ or $\left\{4^{* *}, 2,2, \ldots, 2\right\}$, and $P^{T}$ has column cycle type $\left\{2^{*}, 2^{*}, 2,2, \ldots, 2\right\},\left\{2^{* *}, 2,2, \ldots, 2\right\}$ or $\left\{4^{* *}, 2,2, \ldots, 2\right\}$.

(7) If P satisfies Condition (5.3) then either

(7.1) $n \equiv 5(\bmod 8)$ and $P$ has column cycle type $\left\{5^{* *}, 4,4, \ldots, 4\right\}$; or

(7.2) one of the following holds for $X=P$ and one of the following holds for $X=P^{T}$.

$* n \equiv 3(\bmod 6)$ and $X$ has column cycle type $\left\{3^{*}, 3^{*}, 3,3, \ldots, 3\right\}$.

* $n \equiv 3(\bmod 6)$ and $X$ has column cycle type $\left\{3^{* *}, 3,3, \ldots, 3\right\}$.

$* n \equiv-3(\bmod 2 p)$ and $X$ has column cycle type $\left\{p-3^{* *}, p, p, \ldots, p\right\}$ for some $p \geq 5$.

(8) Row $\alpha$ is in the top unless the cycle type satisfies Condition (5.1), in which case row $\alpha$ may be in the top or in the bottom.

(9) If P satisfies Condition (4.4), (4.5) or (5.3), then row $\beta$ is in the top.

(10) $\alpha, \beta \neq m$.

(11) $\alpha \notin\left\{\sigma_{1}, \sigma_{2}, \ldots, \sigma_{\omega}\right\}$ and if $\beta=\sigma_{i}$ for some $i \in\{1,2, \ldots, \omega\}$, then $\alpha=\beta-1$ and $m_{i}=2$.

(12) If $m_{i}=2^{* *}$ for some $i \in\{1,2, \ldots, \omega\}$, then $\beta \neq m-1$.

(13) If row $\alpha$ is in the top and row $\beta$ is in the bottom, then $\alpha \neq m-1$.

(14) Row $\alpha$ is segregated.

(15) If $n$ is odd, row $\alpha$ is in the bottom and the left of row $\beta$ contains small symbols, then there is no column in the right which contains a large symbol in row $\alpha$ and a large symbol in row $\beta$.

It is a routine but very tedious exercise to show that either $Q$ or $Q^{T}$ can be transformed into a partial Latin square $P \in \mathcal{P}_{n}$ by permuting rows, columns and symbols. We give only an outline of the proof here, and the interested reader is referred to [3] for a complete account.

Lemma 4.3 Let $n \geq 14$ and let $Q \in \mathcal{Q}_{n}$. Either $Q$ or $Q^{T}$ is isotopic to a partial Latin square $P \in \mathcal{P}_{n}$.

Proof: It is clear that we can permute the rows of $Q$ (or $Q^{T}$ ) so that the disjoint cycles of the permutation $\Lambda(Q)$ (or $\Lambda\left(Q^{T}\right)$ ) are grouped together in the resulting partial Latin square $Q^{\prime}$. We refer to a group of rows corresponding to a cycle of $\Lambda$ as a block. The order of the blocks in $Q^{\prime}$ can be interchanged by permuting rows, and rows can be cyclically permuted within blocks, so that the resulting partial Latin square $Q^{\prime \prime}$ satisfies Conditions 
(4)-(13). (In this step, the proof splits into numerous cases based on the column cycle types of $Q$ and $Q^{T}$, and we omit the details). The columns of $Q^{\prime \prime}$ can be permuted so that columns 1 and 2 are filled, and then symbols can be permuted so that column 1 is in natural order. Finally, columns can be permuted so that the resulting partial Latin square $P$ satisfies Conditions (14) and (15) of Definition 4.2. Then $P$ is the desired partial Latin square.

\section{The Segregation Method}

In this section we give an outline of the method which shall be used to prove the main result. The notation introduced in this section shall be used in Section 6 .

For all $n \leq 5$, it is easy to verify that the exceptions stated in Theorem 1.5 are the only partial Latin squares in $\mathcal{Q}_{n}$ which are not completable. It has been verified by computer that for all $n \in\{6,7, \ldots, 13\}$, every partial Latin square in $\mathcal{Q}_{n}$ is completable (see [3]). The remainder of the paper is devoted to proving that for all $n \geq 14$, every partial Latin square in $\mathcal{Q}_{n}$ is completable. In fact, by Lemmas 4.1 and 4.3 , it is sufficient to show that for all $n \geq 14$, every partial Latin square in $\mathcal{P}_{n}$ is completable.

Let $n \geq 14$ and let $P \in \mathcal{P}_{n}$. In Section 6, we shall construct a completion of $P$ by assuming the existence of a completion of each partial Latin square in $\mathcal{Q}_{m}$ and $\mathcal{Q}_{m+1}$ (recall that throughout the paper, $m=\left\lfloor\frac{n}{2}\right\rfloor$ ). The construction splits into numerous cases depending on which of Conditions (4.1)-(4.5) or Conditions (5.1)-(5.3) of Definition 4.2 $P$ satisfies.

The technique for completing $P$ will involve making a number of changes to $P$ to construct a new partial Latin square $S^{\prime}$ which contains the same filled cells as $P$, but is segregated. The partial Latin square $S^{\prime}$ will then be completed to a segregated Latin square $L^{\prime}$ in such a way that a completion of $P$ can be obtained from $L^{\prime}$.

The partial Latin square $S^{\prime}$ will be obtained from $P$ by swapping pairs of symbols which occur in the same row or in the same column as each other. Suppose that $P$ contains the triples $\left(\rho_{1}, I_{1}, x\right)$ and $\left(\rho_{1}, I_{2}, y\right)$ and that $S^{\prime}$ contains the triples $\left(\rho_{1}, I_{1}, y\right)$ and $\left(\rho_{1}, I_{2}, x\right)$. That is, symbols $x$ and $y$ have been swapped in cells $\left(\rho, I_{1}\right)$ and $\left(\rho, I_{2}\right)$. Then we shall ensure that our completion of $S^{\prime}$ contains a $\left(\rho_{1}, I_{1}, \rho_{2}, I_{2}, x, y\right)$-intercalate for some row $\rho_{2}$. Similarly, if symbols $a$ and $b$ in cells $\left(\varrho_{1}, J_{1}\right)$ and $\left(\varrho_{2}, J_{1}\right)$ have been swapped then we shall ensure that the completion of $S^{\prime}$ contains a $\left(\varrho_{1}, J_{1}, \varrho_{2}, J_{2}, a, b\right)$-intercalate for some column $J_{2}$. Switching in these created intercalates in the completion of $S^{\prime}$ will result in a Latin square in which symbols $x$ and $y$ occur in the same cells as those in which they occur in $P$, and hence we obtain a completion of $P$.

Note that column 1 and row $\alpha$ of $P$ are segregated (see Conditions (2) and (14) of Definition 4.2), but that column 2 and row $\beta$ of $P$ need not be. Thus to transform $P$ into a segregated partial Latin square, we will perform a sequence of swaps on the symbols in column 2 and a sequence of swaps on the symbols in row $\beta$ so that the resulting partial Latin square is segregated. Note that swapping symbols in column 2 and in row $\beta$ so that columns 1 and 2 and rows $\alpha$ and $\beta$ are segregated is sufficient to guarantee that the 
resulting partial Latin square is segregated, unless $n$ is odd and rows $\alpha$ and $\beta$ are both in the bottom. In this latter case, we must also ensure that the bottom right contains two distinct large symbols occurring in distinct columns.

A swap of two symbols which is performed so as to segregate column 2 shall be called a $2^{\text {nd }}$ column-segregation swap, and a swap of two symbols performed so as to segregate row $\beta$ shall be referred to as a $\beta$-segregation swap. Our completion of the segregated square shall contain the necessary intercalates so that we can "undo" the $2^{\text {nd }}$ column-segregation swaps and $\beta$-segregation swaps and hence obtain a completion of the original partial Latin square.

We require that the cells in intercalates which are used to undo $2^{\text {nd }}$ column-segregation swaps are not also used to undo $\beta$-segregation swaps. Thus, we define column-reserved symbols to be symbols involved in $2^{\text {nd }}$ column-segregation swaps and reserved rows to be the rows in which column-reserved symbols occur in column 2.

To guarantee the independence of $2^{\text {nd }}$ column-segregation swaps and $\beta$-segregation swaps, we shall ensure that all but at most one of the $\beta$-segregation swaps are undone by switching in intercalates involving row $\beta$ and only one other row, $\gamma$ say, for some unreserved row $\gamma$. When one $\beta$-segregation swap is undone by switching in an intercalate which does not involve row $\gamma$, we denote by $\gamma^{\prime}$ the row (other than row $\beta$ ) involved in this intercalate.

We reserve the following notation to identify those symbols in row $\beta$ of $P$ which are on the wrong, in terms of $P$ being segregated, side of row $\beta$.

If row $\beta$ is in the top, let $k$ be the number of large symbols in the left of row $\beta$ in $P$, and if row $\beta$ is in the bottom, let $k$ be the number of small symbols in the left of row $\beta$ in $P$. Since $P \in \mathcal{P}_{n}, \beta \neq m$ (see Condition (10) of Definition 4.2). Furthermore, if $\beta=\sigma_{i}$ for some $i \in\{1,2, \ldots, \omega\}$ then $m_{i}=2$ and $\alpha=\beta-1$, and hence $\beta \neq m+1$ (see Conditions (10), (11) and (12) of Definition 4.2). Thus none of these $k$ symbols occur in columns 1 or 2 of $P$, and so $0 \leq k \leq m-2$. We will denote these $k$ symbols by $x_{1}, x_{2}, \ldots, x_{k}$ and denote the columns containing them by $C_{1}, C_{2}, \ldots, C_{k}$. That is, $\left(\beta, C_{i}, x_{i}\right) \in P$ for $i=1,2, \ldots, k$.

It follows that there are $k$ small symbols in the right of row $\beta$ when row $\beta$ is in the top, that there are $k$ large symbols in the right of row $\beta$ when row $\beta$ is in the bottom and $n$ is even, and that there are $k+1$ large symbols in the right of row $\beta$ when row $\beta$ is in the bottom and $n$ is odd. We will denote these $k$ or $k+1$ symbols by $y_{1}, y_{2}, \ldots, y_{k}$ or $y_{1}, y_{2}, \ldots, y_{k+1}$ and denote the columns containing them by $D_{1}, D_{2}, \ldots, D_{k}$ or $D_{1}, D_{2}, \ldots, D_{k+1}$ respectively. That is, $\left(\beta, D_{i}, y_{i}\right) \in P$ for $i=1,2, \ldots, k$, or for $i=$ $1,2, \ldots, k+1$ if row $\beta$ is in the bottom and $n$ is odd.

The swapping graph of $P$ is the bipartite graph $G$ which we now define. If $n$ is odd and row $\beta$ is in the bottom then $G$ has partite sets $V_{1}=\left\{x_{1}, x_{2}, \ldots, x_{k}\right\}$ and $V_{2}=\left\{y_{1}, y_{2}, \ldots, y_{k+1}\right\}$. Otherwise, $G$ has partite sets $V_{1}=\left\{x_{1}, x_{2}, \ldots, x_{k}\right\}$ and $V_{2}=$ $\left\{y_{1}, y_{2}, \ldots, y_{k}\right\}$. The edge set of $G$ is given by $x_{i} y_{j} \in E(G)$ if and only if $x_{i}$ and $y_{j}$ are swappable. Note that symbols $x_{i}$ and $y_{j}$ are swappable unless $\left(\alpha, C_{i}, y_{j}\right) \in P$ or $\left(\alpha, D_{j}, x_{i}\right) \in P$ and thus we have the following lemma. 
Lemma 5.1 Let $P \in \mathcal{P}_{n}$. Let $G$ be the swapping graph of $P$ and let the partite sets of $G$ be $V_{1}$ and $V_{2}$. The degree of any vertex in $V_{2}$ is at least $\left|V_{1}\right|-2$ and the degree of any vertex in $V_{1}$ is at least $\left|V_{2}\right|-2$.

It is clear that if $G$ contains a matching that includes $V_{1}$ then we can segregate row $\beta$ by swapping the pairs of matched symbols in $G$.

There are two complicating factors in the process of segregating row $\beta$. Firstly, if row $\alpha$ and row $\beta$ are both in the top, or both in the bottom, then $G$ does not necessarily contain a matching that includes $V_{1}$. We will see examples of this shortly. Secondly, if row $\alpha$ is in the top and row $\beta$ is in the bottom then some care needs to be taken in choosing this matching that includes $V_{1}$ (so that, for example, the resulting segregated partial Latin square can be completed to a segregated Latin square, and so that cells later involved in undoing $\beta$-segregation swaps are not also involved in $2^{\text {nd }}$ column-segregation swaps).

Suppose that row $\alpha$ of $P$ is in the top and row $\beta$ of $P$ is in the bottom. Since row $\alpha$ is segregated, it is clear that a small symbol in the left of row $\beta$ can be swapped with any large symbol in the right of row $\beta$. Thus, $G=K_{k, k}$ when $n$ is even and $G=K_{k, k+1}$ when $n$ is odd, and so $G$ contains a matching that includes $V_{1}$. In this situation the second complicating factor arises: certain choices of the matching that includes $V_{1}$ gives rise to problems in later steps of the completion.

On the other hand, if rows $\alpha$ and $\beta$ are both in the top, or both in the bottom, then $G$ does not necessarily contain a matching that includes $V_{1}$. When $G$ does not contain a matching that includes $V_{1}$ we actually begin by swapping some symbols in row $\beta$ so that row $\beta$ becomes less segregated. That is, by performing these swaps we increase the number of symbols in row $\beta$ of $P$ which are on the wrong, in terms of $P$ being segregated, side of row $\beta$. However, these swaps are chosen in such a way that the swapping graph, $G^{*}$ say, (with partite sets $V_{1}^{*}$ and $V_{2}^{*}$ ) of the resulting partial Latin square contains a matching that includes $V_{1}^{*}$. Swaps which are performed so as to ensure that $G^{*}$ contains a matching that includes $V_{1}^{*}$ shall be referred to as dummy swaps.

We now establish more specific conditions on when $G$ contains a matching that includes $V_{1}$. This result will be used in Section 6 .

Lemma 5.2 Let $P \in \mathcal{P}_{n}$ with filled rows $\alpha$ and $\beta$ in the top. Let $G$ be the swapping graph of $P$ and let $k$ be the number of large symbols in the left of row $\beta$. If $k \geq 4$ then $G$ contains a perfect matching.

Proof: By Lemma 5.1, the minimum degree of $G$ is at least $k-2$. Since $k \geq 4$, we have minimum degree at least $\frac{k}{2}$ and so by Lemma 3.3, $G$ contains a perfect matching.

The following three examples illustrate that if rows $\alpha$ and $\beta$ are in the top then $G$ does not necessarily contain a perfect matching when $k \in\{1,2,3\}$. In these examples we have $n=14, m=7, \alpha=1$ and $\beta=2$. In each we give only rows $\alpha$ and $\beta$.

(1) $k=1, x_{1}=9, C_{1}=6, y_{1}=4, D_{1}=11$.

\begin{tabular}{|c|c|c|c|c|c|c||c|c|c|c|c|c|c|}
\hline 1 & 2 & 4 & 6 & 3 & 5 & 7 & 10 & 12 & 8 & 9 & 11 & 13 & 14 \\
\hline 2 & 3 & 1 & 5 & 7 & 9 & 6 & 11 & 13 & 12 & 4 & 10 & 14 & 8 \\
\hline
\end{tabular}


(2) $k=2, x_{1}=9, C_{1}=4, x_{2}=10, C_{2}=5, y_{1}=4, D_{1}=8, y_{2}=5, D_{2}=9$.

\begin{tabular}{|c|c|c|c|c|c|c||c|c|c|c|c|c|c|}
\hline 1 & 2 & 6 & 5 & 4 & 3 & 7 & 9 & 10 & 14 & 12 & 8 & 11 & 13 \\
\hline 2 & 3 & 1 & 9 & 10 & 7 & 6 & 4 & 5 & 13 & 8 & 12 & 14 & 11 \\
\hline
\end{tabular}

(3) $k=3, x_{1}=12, C_{1}=4, x_{2}=8, C_{2}=6, x_{3}=9, C_{3}=7, y_{1}=5, D_{1}=8, y_{2}=4, D_{2}=$ $11, y_{3}=1, D_{3}=12$.

\begin{tabular}{|c|c|c|c|c|c|c||c|c|c|c|c|c|c|}
\hline 1 & 2 & 3 & 6 & 7 & 4 & 5 & 8 & 11 & 12 & 9 & 14 & 10 & 13 \\
\hline 2 & 3 & 7 & 12 & 6 & 8 & 9 & 5 & 10 & 13 & 4 & 1 & 11 & 14 \\
\hline
\end{tabular}

\section{Main Construction}

Let $P \in \mathcal{P}_{n}$ be a partial Latin square of order $n \geq 14$. In Lemma 6.1 we construct a completion of $P$ under the assumption that every partial Latin square in $\mathcal{Q}_{m} \cup \mathcal{Q}_{m+1}$ is completable, thus setting up a proof by induction of our main result.

Lemma 6.1 Let $n \geq 14$, let $m=\left\lfloor\frac{n}{2}\right\rfloor$ and let $P \in \mathcal{P}_{n}$. If each $Q \in \mathcal{Q}_{m} \cup \mathcal{Q}_{m+1}$ is completable, then $P$ is completable.

Proof: Recall from Definition 4.2 that $P$ has column cycle type $\left\{m_{1}, m_{2}, \ldots, m_{\omega}\right\}$ for some $m_{i}, i=1,2, \ldots, \omega$ and $P$ satisfies at least one of Conditions (4.1) - (4.5) and $(5.1)-(5.3)$. The proof is divided into cases depending on which of Conditions (4.1)-(4.5) or (5.1)-(5.3) $P$ satisfies. The constructions for each case are all quite similar. However, there are subtle technical differences in the details of the arguments, and these differences make it necessary to consider each of the cases individually. For reasons of brevity we include here only the construction for the cases where $P$ satisfies Condition (4.2) or Condition (5.2) of Definition 4.2. The proofs for the other cases are quite similar and can be found in [3].

Thus, we shall assume that $P$ has column cycle type $\left\{m_{1}, m_{2}, \ldots, m_{\omega}\right\}$ such that $\sigma_{r-1} \leq m-2$ and $\sigma_{r} \geq m+2$ for some $r \in\{1,2, \ldots, \omega\}$, where $\sigma_{0}=0$ and $\sigma_{i}=$ $m_{1}+m_{2}+\ldots+m_{i}$ for $i=1,2, \ldots, \omega$. Recall from Section 5 that the first step of our construction is to transform $P$ into a segregated partial Latin square. We begin by segregating column 2 of $P$.

Let $P^{\prime}$ be the partial Latin square obtained from $P$ by swapping symbols $m+1$ and $\sigma_{r-1}+1$ in cells $(m, 2)$ and $\left(\sigma_{r}, 2\right)$. Then columns 1 and 2 and row $\alpha$ of $P^{\prime}$ are segregated. We shall construct a completion of $P^{\prime}$ which contains an $\left(m, 2, \sigma_{r}, C, \sigma_{r-1}+1, m+1\right)$ intercalate for some column $C$, so that a completion of $P$ can be obtained by switching in this intercalate. The construction now splits into two cases.

- Case 1: Filled rows $\alpha$ and $\beta$ are in the top.

- Case 2: Filled row $\alpha$ is in the top and filled row $\beta$ is in the bottom. 


\section{Case 1: Filled rows $\alpha$ and $\beta$ are in the top.}

We will now segregate row $\beta$ of $P^{\prime}$, if it is not already segregated. Let $k$ be the number of large symbols in the left of row $\beta$, let $G$ be the swapping graph of $P^{\prime}$ and let $C_{1}, C_{2}, \ldots, C_{k}$, $D_{1}, D_{2}, \ldots, D_{k}, x_{1}, x_{2}, \ldots, x_{k}$ and $y_{1}, y_{2}, \ldots, y_{k}$ be as defined in Section 5 (see page 12). By Lemma 5.2, we have that $G$ contains a perfect matching when $k \geq 4$. However, if $k \in\{1,2,3\}$ then $G$ does not necessarily contain a perfect matching. In this case we perform dummy swaps to ensure that the swapping graph of the resulting partial Latin square contains a perfect matching.

We now explicitly describe these dummy swaps, for the cases $k=1, k=2$ and $k=3$ where $G$ does not contain a perfect matching. First we select the cells in which the dummy swaps will occur.

(i) $k=1$ : We select two filled cells in row $\beta,\left(\beta, W_{1}, s_{1}\right)$ and $\left(\beta, Z_{1}, t_{1}\right)$ say.

(ii) $k=2$ and $E(G)=\emptyset$ : We select four filled cells in row $\beta,\left(\beta, W_{1}, s_{1}\right),\left(\beta, W_{2}, s_{2}\right)$, $\left(\beta, Z_{1}, t_{1}\right)$ and $\left(\beta, Z_{2}, t_{2}\right)$ say.

(iii) $k=2$ and $|E(G)| \geq 1$ : Without loss of generality, $x_{2} y_{2} \in E(G)$. We select two filled cells in row $\beta,\left(\beta, W_{1}, s_{1}\right)$ and $\left(\beta, Z_{1}, t_{1}\right)$ say.

(iv) $k=3$ : We select two filled cells in row $\beta,\left(\beta, W_{1}, s_{1}\right)$ and $\left(\beta, Z_{1}, t_{1}\right)$ say.

Let $D$ be the set of subscripts of these dummy swaps; that is, $D=\{1\}$ if Condition (i), (iii) or (iv) is satisfied, $D=\{1,2\}$ if Condition (ii) is satisfied, and $D=\emptyset$ if $k=0$ or if $G$ contains a perfect matching. Let $S$ be the partial Latin square obtained from $P^{\prime}$ by replacing the triples $\left(\beta, W_{i}, s_{i}\right)$ and $\left(\beta, Z_{i}, t_{i}\right)$ with $\left(\beta, W_{i}, t_{i}\right)$ and $\left(\beta, Z_{i}, s_{i}\right)$ for each $i \in D$.

We place restrictions on our choice of $W_{i}, Z_{i}, s_{i}$ and $t_{i}$ for $i \in D$, to ensure that the swapping graph $G^{*}$ of $S$ contains a perfect matching. For each $i \in D$, we require that:

- $W_{i} \in\{3,4, \ldots, m\} \backslash\left\{C_{1}, C_{2}, \ldots, C_{k}\right\}$ (so $s_{i}$ is small with $s_{i} \notin\left\{y_{1}, y_{2}, \ldots, y_{k}\right\}$ );

- $Z_{i} \in\{m+1, m+2, \ldots, n\} \backslash\left\{D_{1}, D_{2}, \ldots, D_{k}\right\}$ (so $t_{i}$ is large with $t_{i} \notin\left\{x_{1}, x_{2}, \ldots, x_{k}\right\}$ ); and

- $t_{i} \neq m+1$ (since $m+1$ is a column-reserved symbol).

Note that if $P^{\prime}$ satisfies Condition (ii) or (iv) above, then the number of large symbols in the left of row $\beta$ of $S$ is four. Hence by Lemma 5.2, any choice of $W_{i}$ and $Z_{i}$ for $i \in D$ satisfying these requirements will result in $G^{*}$ containing a perfect matching. Furthermore, since $m \geq 7$ it is easy to check that suitable choices exist for $W_{i}, Z_{i}, s_{i}$ and $t_{i}$, for each $i \in D$. However, if $P^{\prime}$ satisfies Condition (i) or (iii), some additional care must be taken in choosing $W_{1}, Z_{1}, s_{1}$ and $t_{1}$ to ensure that $G^{*}$ contains a perfect matching. In these cases, we also require that:

- symbol $s_{1}$ is not in cell $\left(\alpha, C_{1}\right)$;

- symbol $t_{1}$ is not in cell $\left(\alpha, D_{1}\right)$; 
- symbol $x_{1}$ is not in cell $\left(\alpha, Z_{1}\right)$; and

- symbol $y_{1}$ is not in cell $\left(\alpha, W_{1}\right)$.

These additional restrictions ensure that the edges $s_{1} x_{1}$ and $t_{1} y_{1}$ are in $E\left(G^{*}\right)$. These edges, along with $x_{2} y_{2}$ in the case where $P^{\prime}$ satisfies Condition (iii), form a perfect matching in $G^{*}$.

We need to check that suitable choices for $W_{1}, Z_{1}, s_{1}$ and $t_{1}$ exist when $P^{\prime}$ satisfies Condition (i) or (iii). If $P^{\prime}$ satisfies Condition (i), simple counting arguments give that there are at least $m-5$ columns from which to choose $W_{1}$ and at least $m-4$ columns from which to choose $Z_{1}$. If $P^{\prime}$ satisfies Condition (iii), simple counting arguments give that there are at least $m-6$ columns from which to choose $W_{1}$ and at least $m-5$ columns from which to choose $Z_{1}$. Since $m \geq 7$, suitable choices exist in both cases.

Let $k^{\prime}=k+|D|$. For brevity, we introduce the set $B=\left\{1,2, \ldots, k^{\prime}\right\}$ with $B=\emptyset$ when $k^{\prime}=0$. We relabel the large symbols in the left of row $\beta$ in $S$ with $x_{1}, x_{2}, \ldots, x_{k^{\prime}}$ and their corresponding columns with $C_{1}, C_{2}, \ldots, C_{k^{\prime}}$. We relabel the small symbols in the right of row $\beta$ in $S$ with $y_{1}, y_{2}, \ldots, y_{k^{\prime}}$ and their corresponding columns with $D_{1}, D_{2}, \ldots, D_{k^{\prime}}$, such that $x_{i}$ is matched with $y_{i}$ for each $i \in B$ in the perfect matching in $G^{*}$. Let $S^{\prime}$ be the segregated partial Latin square obtained from $S$ by replacing the triples $\left(\beta, C_{i}, x_{i}\right)$ and $\left(\beta, D_{i}, y_{i}\right)$ with $\left(\beta, C_{i}, y_{i}\right)$ and $\left(\beta, D_{i}, x_{i}\right)$ for each $i \in B$. Recall from Section 5 that a swap of two symbols $x_{i}$ and $y_{i}$, performed so as to segregate row $\beta$, shall be referred to as a $\beta$-segregation swap.

We shall now begin constructing a segregated completion $L^{\prime}$ of $S^{\prime}$ in such a way that we can transform $L^{\prime}$ into a completion of $P$. To do this, we shall need to be able to undo (in order) the following swaps:

(1) the $\beta$-segregation swaps (if any) made in transforming $S$ into $S^{\prime}$;

(2) the dummy swaps (if any) made in transforming $P^{\prime}$ into $S$; and

(3) the swap of $m+1$ and $\sigma_{r-1}+1$ made in transforming $P$ into $P^{\prime}$.

To do this, we shall ensure that $L^{\prime}$ contains an intercalate containing symbols $x_{i}$ and $y_{i}$ for each $i \in B$, an intercalate containing symbols $s_{j}$ and $t_{j}$ for each $j \in D$ and an intercalate containing symbols $m+1$ and $\sigma_{r-1}+1$ such that a completion of $P$ is obtained by switching in each of these intercalates. Actually, not all of these intercalates exist in $L^{\prime}$; some of them arise only after switching in others.

By the inductive assumption, we can complete the top left of $S^{\prime}$ with small symbols. Do this and let the resulting partial Latin square be $Q_{1}$.

We require our completion of $S^{\prime}$ to contain an intercalate containing symbols $x_{i}$ and $y_{i}$ for each $i \in B$. Recall from Section 5 that all but at most one $\beta$-segregation swap will be undone by switching in an intercalate involving row $\gamma$, for some unreserved row $\gamma$ in the bottom. More specifically, we show that we can find a row $\gamma$ with $\gamma \neq \sigma_{r}$ in the bottom of $Q_{1}$ such that we can place symbol $x_{i}$ in cell $\left(\gamma, C_{i}\right)$ for at least $k^{\prime}-1$ values of $i \in B$. Note that the only possible constraint on placing symbol $x_{i}$ in cell $\left(\gamma, C_{i}\right)$ is the occurrence of symbol $x_{i}$ in cell $(\gamma, 1)$ or cell $(\gamma, 2)$. 
Let $T=\{m+1, m+2, \ldots, n\} \backslash\left\{x_{1}, x_{2}, \ldots, x_{k^{\prime}}\right\}$ and note that $|T| \geq 2$. Since $Q_{1}$ is segregated, each symbol in $T$ occurs in the bottom of column 1 and in the bottom of column 2 in $Q_{1}$. We choose row $\gamma$ with $\gamma \neq \sigma_{r}$ such that the symbol in cell $(\gamma, 1)$ or cell $(\gamma, 2)$ of $Q_{1}$ is in $T$. Then row $\gamma$ has the desirable property that we can place symbol $x_{i}$ in cell $\left(\gamma, C_{i}\right)$ for at least $k^{\prime}-1$ values of $i \in B$. If we can place symbol $x_{i}$ in cell $\left(\gamma, C_{i}\right)$ for all $i \in B$, then we shall say that row $\gamma$ sets up all switches. If row $\gamma$ does not set up all switches, we shall denote by $x_{l}$ the unique symbol in $\left\{x_{1}, x_{2}, \ldots, x_{k^{\prime}}\right\}$ which cannot be placed in row $\gamma$ of $Q_{1}$. Let

$$
Q_{2}=\left\{\begin{array}{l}
Q_{1} \cup\left\{\left(\gamma, C_{i}, x_{i}\right),\left(\gamma, D_{i}, y_{i}\right): i \in B\right\} \text { if } B \neq \emptyset \text { and row } \gamma \text { sets up all switches; } \\
Q_{1} \cup\left\{\left(\gamma, C_{i}, x_{i}\right),\left(\gamma, D_{i}, y_{i}\right): i \in B \backslash\{l\}\right\} \text { if } B \neq \emptyset \text { and row } \gamma \text { does not set up } \\
\quad \text { all switches; } \\
Q_{1} \text { otherwise. }
\end{array}\right.
$$

If $D=\emptyset$, we let $Q_{3}=Q_{2}$. Otherwise, we ensure that for each $j \in D$, our completion of $S^{\prime}$ will contain a $\left(\beta, W_{j}, \epsilon_{j}, Z_{j}, s_{j}, t_{j}\right)$-intercalate for some row $\epsilon_{j}$ in the top. Note that cells $\left(\beta, W_{j}\right)$ and $\left(\beta, Z_{j}\right)$ of $Q_{2}$ do not contain symbols $t_{j}$ and $s_{j}$. However, the $\beta$ segregation swaps made in transforming $S$ into $S^{\prime}$ will be undone before we switch in the $\left(\beta, W_{j}, \epsilon_{j}, Z_{j}, s_{j}, t_{j}\right)$-intercalate. Hence, when it comes time to undo the swap of $s_{j}$ and $t_{j}$, we will have $\left(\beta, W_{j}, t_{j}\right)$ and $\left(\beta, Z_{j}, s_{j}\right)$ in our Latin square. For each $j \in D$, let $\epsilon_{j}$ be the row of $Q_{2}$ in which symbol $s_{j}$ occurs in column $W_{j}$ and let $Q_{3}=Q_{2} \cup\left\{\left(\epsilon_{j}, Z_{j}, t_{j}\right): j \in D\right\}$.

To complete the construction we consider separately the case $n$ is even and the case $n$ is odd.

The case $n$ is even for rows $\alpha$ and $\beta$ in the top: If $B=\emptyset$, we let $Q_{4}=Q_{3}$. Otherwise, we let $Q_{4}$ be the partial Latin square obtained from $Q_{3}$ by completing the left of row $\gamma$ with large symbols.

Unless $B \neq \emptyset$ and row $\gamma$ does not set up all switches, we let $Q_{5}=Q_{4}$. If $B \neq \emptyset$ and row $\gamma$ does not set up all switches, we must ensure that our completion of $S^{\prime}$ contains an intercalate containing symbols $x_{l}$ and $y_{l}$. To do this, we choose some row, $\gamma^{\prime}$ say, with $\gamma^{\prime} \neq \sigma_{r}$ in the bottom of $Q_{4}$ such that cell $\left(\gamma^{\prime}, C_{l}\right)$ is allowable for symbol $x_{l}$, and let $Q_{5}=Q_{4} \cup\left\{\left(\gamma^{\prime}, C_{l}, x_{l}\right),\left(\gamma^{\prime}, D_{l}, y_{l}\right)\right\}$.

We now fill the empty cells in the bottom left of $Q_{5}$ with large symbols to form the partial Latin square $Q_{6}$. If either $B=\emptyset$ or $B \neq \emptyset$ and row $\gamma$ sets up all switches, then Theorem 1.3 or Lemma 3.1 give the required completion of the bottom left. Otherwise, $B \neq \emptyset$ and row $\gamma$ does not set up all switches, and we first apply Lemma 3.4 to complete the left of row $\gamma^{\prime}$ in $Q_{5}$ with large symbols. By the inductive assumption, the bottom left of the resulting partial Latin square can then be completed with large symbols.

We now ensure that our completion will contain an $\left(m, 2, \sigma_{r}, C, m+1, \sigma_{r-1}+1\right)$ intercalate for some $C$. We choose our column $C$ in the right of $Q_{6}$ such that cell $(m, C)$ is allowable for symbol $m+1$ and cell $\left(\sigma_{r}, C\right)$ is allowable for symbol $\sigma_{r-1}+1$. To verify the existence of such a column, we note that there are precisely two occurrences of symbol $m+1$ in the top right (in rows $\alpha$ and $\beta$, noting that $t_{i} \neq m+1$ for each $i \in D$ ) and there is at most one occurrence of symbol $\sigma_{r-1}+1$ in the bottom right (possibly in row $\gamma$ or row $\gamma^{\prime}$ ). The right of row $\sigma_{r}$ is empty and there are at most two filled cells in 
row $m$ (namely $\left(\epsilon_{i}, Z_{i}, t_{i}\right)$ for $\left.i \in D\right)$. Thus there are at least $m-5$ choices for $C$. Let $Q_{7}=Q_{6} \cup\left\{(m, C, m+1),\left(\sigma_{r}, C, \sigma_{r-1}+1\right)\right\}$.

The top right of $Q_{7}$ contains two filled rows and at most three other filled cells (namely $\left(\epsilon_{i}, Z_{i}, t_{i}\right)$ for each $i \in D$ and $\left.(m, C, m+1)\right)$. Thus we may apply Lemma 3.8 to complete the top right of $Q_{7}$ with large symbols. Denote the resulting partial Latin square by $Q_{8}$.

To embed $Q_{8}$ in a completion $L^{\prime}$ of $S^{\prime}$, it remains only to fill the empty cells in the bottom right. The bottom right of $Q_{8}$ contains at most $m-1$ symbols, all of which are small. Thus we may apply Theorem 1.1 to complete the bottom right of $Q_{8}$ with small symbols.

The case $n$ is odd for rows $\alpha$ and $\beta$ in the top: This case is similar to the case $n$ is even for rows $\alpha$ and $\beta$ in the top, except that here we need to place $m+1$ distinct large symbols in the bottom right (see page 5 ). To do this, we make use of the $2 m+2$ appended cells $(n+1, m+1),(n+1, m+2), \ldots,(n+1, n)$ and $(m+1, n+1),(m+2, n+1)$, $\ldots,(n, n+1)$.

To begin, if $B=\emptyset$ we let $Q_{4}=Q_{3}$. Otherwise, we let $Q_{4}$ be the partial Latin square obtained from $Q_{3}$ by completing the augmented left of row $\gamma$ with large symbols.

Unless $B \neq \emptyset$ and row $\gamma$ does not set up all switches, we let $Q_{5}=Q_{4}$. If $B \neq \emptyset$ and row $\gamma$ does not set up all switches, we must ensure that our completion of $S^{\prime}$ contains an intercalate containing symbols $x_{l}$ and $y_{l}$. To do this, we choose some row, $\gamma^{\prime}$ say, with $\gamma^{\prime} \neq \sigma_{r}$ in the bottom of $Q_{4}$ such that cell $\left(\gamma^{\prime}, C_{l}\right)$ is allowable for symbol $x_{l}$, and let $Q_{5}=Q_{4} \cup\left\{\left(\gamma^{\prime}, C_{l}, x_{l}\right),\left(\gamma^{\prime}, D_{l}, y_{l}\right)\right\}$.

We now fill the remaining empty cells of the augmented bottom left of $Q_{5}$ to form the partial Latin square $Q_{6}$. If either $B=\emptyset$ or $B \neq \emptyset$ and row $\gamma$ sets up all switches, then Theorem 1.3 or Lemma 3.1 give the required completion of the augmented bottom left. Otherwise, $B \neq \emptyset$ and row $\gamma$ does not set up all switches, and we first apply Lemma 3.4 to complete the augmented left of row $\gamma^{\prime}$ of $Q_{5}$ with large symbols. By the inductive assumption, the augmented bottom left of the resulting partial Latin square can then be completed with large symbols.

Recall from Section 2 that in a segregated Latin square of odd order, the bottom right contains $m+1$ distinct large symbols, no two of which occur in the same row or the same column. In particular, our segregated completion of $S^{\prime}$ must satisfy this property. To begin the process of placing these large symbols in the bottom right, consider row $\sigma_{r}$ and the rows (if any) in the bottom of $Q_{6}$ which contain one or more filled cells in the right. (There are at most three such rows, namely rows $\sigma_{r}, \gamma$ and $\gamma^{\prime}$.) Let $r_{1}$ be the symbol in the appended cell $\left(\sigma_{r}, n+1\right)$. If $B \neq \emptyset$, let $r_{2}$ be the symbol in the appended cell $(\gamma, n+1)$. If $B \neq \emptyset$ and row $\gamma$ does not set up all switches, let $r_{3}$ be the symbol in the appended cell $\left(\gamma^{\prime}, n+1\right)$.

If $B \neq \emptyset$, we choose a column, $H_{2}$ say, in the right for which cell $\left(\gamma, H_{2}\right)$ is allowable for symbol $r_{2}$. To verify the existence of such a column, we note that there are at most $m-2$ filled cells in the right of row $\gamma$. There are precisely two occurrences of symbol $r_{2}$ in the top right, in rows $\alpha$ and $\beta$. Note that $r_{2} \neq t_{i}$ for any $i \in D$, since each symbol in $\left\{x_{1}, x_{2}, \ldots, x_{k^{\prime}}\right\}$ (including $t_{i}$ ) occurs in the left of row $\gamma$ (either in cell $(\gamma, 1)$, cell $(\gamma, 2)$ or cell $\left(\gamma, C_{j}\right)$ for some $\left.j \in B\right)$. Thus there is at least $(m+1)-(m-2)-2=1$ such 
column.

Similarly, if $B \neq \emptyset$ and row $\gamma$ does not set up all switches, we choose a column, $H_{3}$ say, which is in the right, distinct from column $H_{2}$, and for which cell $\left(\gamma^{\prime}, H_{3}\right)$ is allowable for symbol $r_{3}$. To verify the existence of such a column, we note that there is precisely one filled cell in the right of row $\gamma^{\prime}$ and at most three occurrences of symbol $r_{3}$ in the top right (in rows $\alpha$ and $\beta$ and possibly in cell $\left(\epsilon_{i}, Z_{i}\right)$ for at most one $i \in D$ ). Thus, given the further requirement that $H_{3} \neq H_{2}$, there are at least $m-4$ choices for $H_{3}$.

We now choose a column, $H_{1}$ say, which is in the right, distinct from columns $H_{2}$ and $H_{3}$, and for which cell $\left(\sigma_{r}, H_{1}\right)$ is allowable for symbol $r_{1}$. There are at most three occurrences of symbol $r_{1}$ in the top right (in rows $\alpha$ and $\beta$ and possibly in cell $\left(\epsilon_{i}, Z_{i}\right)$ for at most one $i \in D)$. The right of row $\sigma_{r}$ is empty. Thus there are at least $m-4$ choices for $H_{1}$. We place symbol $r_{1}$ in the appended cell $\left(n+1, H_{1}\right)$, symbol $r_{2}$ in the appended cell $\left(n+1, H_{2}\right)$ (when $B \neq \emptyset$ ) and symbol $r_{3}$ in the appended cell $\left(n+1, H_{3}\right)$ (when $B \neq \emptyset$ and row $\gamma$ does not set up all switches). We let

$$
Q_{7}=\left\{\begin{aligned}
Q_{6} \cup\left\{\left(\sigma_{r}, H_{1}, r_{1}\right),\left(\gamma, H_{2}, r_{2}\right)\right\} \text { if } B \neq \emptyset \text { and row } \gamma \text { sets up all switches; } \\
Q_{6} \cup\left\{\left(\sigma_{r}, H_{1}, r_{1}\right),\left(\gamma, H_{2}, r_{2}\right),\left(\gamma^{\prime}, H_{3}, r_{3}\right)\right\} \text { if } B \neq \emptyset \text { and row } \gamma \text { does not set up } \\
\quad \text { all switches; } \\
Q_{6} \cup\left\{\left(\sigma_{r}, H_{1}, r_{1}\right)\right\} \text { otherwise. }
\end{aligned}\right.
$$

The augmented top right of $Q_{7}$ consists of the two filled rows $\alpha$ and $\beta$, the partially filled row $n+1$ containing at most three filled cells, and at most two other filled cells (namely $\left(\epsilon_{i}, Z_{i}, t_{i}\right)$ for each $\left.i \in D\right)$ which contain distinct symbols and occur in distinct columns. Thus we may apply Lemma 3.6 to complete (with large symbols) row $n+1$ of the augmented top right of $Q_{7}$.

We next ensure that our completion will contain an $\left(m, 2, \sigma_{r}, C, m+1, \sigma_{r-1}+1\right)$ intercalate for some column $C$. We choose our column $C$ in the right of $Q_{7}$ such that cell $(m, C)$ is allowable for symbol $m+1$ and cell $\left(\sigma_{r}, C\right)$ is allowable for symbol $\sigma_{r-1}+1$. To verify the existence of such a column, we note that there are precisely three occurrences of symbol $m+1$ in the augmented top right of $Q_{7}$ (in rows $\alpha, \beta$ and $n+1$, noting that $t_{i} \neq m+1$ for any $\left.i \in D\right)$ and at most one occurrence of symbol $\sigma_{r-1}+1$ in the bottom right of $Q_{7}$ (possibly in row $\gamma$ or row $\gamma^{\prime}$ ). There are at most two filled cells in the right of row $m+1$ (namely $\left(\epsilon_{i}, Z_{i}, t_{i}\right)$ for $i \in D$ ) and there is precisely one filled cell in the right of row $\sigma_{r}$ (namely $\left(\sigma_{r}, H_{1}, r_{1}\right)$ ). Thus there are at least $m-6$ choices for $C$.

There is one situation in which additional care needs to be taken in choosing the column $C$. Suppose that $n=8,|D|=2$ and that $\epsilon_{1}=\epsilon_{2}=m$; that is, each dummy swap is to be undone via switching in an intercalate involving row $m$. Further suppose that the augmented top right of $Q_{7}$ contains a 3 by 3 subsquare based on symbols from the set $\{1,2, \ldots, n\} \backslash\left\{t_{1}, t_{2}, m+1\right\}$, in rows $\alpha, \beta$ and $n+1$ and in columns $I_{1}, I_{2}$ and $I_{3}$ say, such that $I_{1}, I_{2}, I_{3} \notin\left\{\epsilon_{1}, \epsilon_{2}\right\}$. It is clear that the three cells $\left(m, I_{1}\right),\left(m, I_{2}\right)$ and $\left(m, I_{3}\right)$ are allowable for symbol $m+1$. Furthermore, at least one of the cells $\left(\sigma_{r}, I_{1}\right),\left(\sigma_{r}, I_{2}\right)$ and $\left(\sigma_{r}, I_{3}\right)$ is allowable for symbol $\sigma_{r-1}+1$. We choose $C$ from $\left\{I_{1}, I_{2}, I_{3}\right\}$ such that cell $(m, C)$ is allowable for symbol $m+1$ and cell $\left(\sigma_{r}, C\right)$ is allowable for symbol $\sigma_{r-1}+1$. 
Let $Q_{8}=Q_{7} \cup\left\{(m, C, m+1),\left(\sigma_{r}, C, \sigma_{r-1}+1\right)\right\}$. We now complete the augmented top right of $Q_{8}$ with large symbols. The augmented top right of $Q_{8}$ contains three filled rows and at most three other filled cells (namely $\left(\epsilon_{i}, Z_{i}, t_{i}\right)$ for $i \in D$ and $(m, C, m+1)$ ). Lemma 3.7 and our choice of column $C$ guarantee that the augmented top right of $Q_{8}$ can be completed. We denote the resulting partial Latin square by $Q_{9}$.

If $B=\emptyset$, we let $Q_{10}=Q_{9}$. Otherwise, we now complete the right of row $\gamma$ of $Q_{9}$ with small symbols. If row $\gamma$ does not set up all switches and cell $\left(\gamma, D_{l}\right)$ is empty, then there are at most $m-3$ small symbols in row $\gamma$ and at most two small symbols in column $D_{l}$ (in cells $\left(\gamma^{\prime}, D_{l}\right)$ and possibly in $(m+1, C)$ ). We fill cell $\left(\gamma, D_{l}\right)$ with one of at least $m-(m-3)-2=1$ allowable small symbols. If cell $(\gamma, C)$ now remains empty, then there are at most $m-2$ small symbols in row $\gamma$ and precisely one small symbol in column $C$. We fill cell $(\gamma, C)$ with one of at least $m-(m-2)-1=1$ allowable small symbols. Then any remaining empty cells in row $\gamma$ may be filled without restriction using the remaining small symbols not occurring in row $\gamma$. Do this and let the resulting partial Latin square be $Q_{10}$.

To embed $Q_{10}$ in a completion $L^{\prime}$ of $S^{\prime}$, it remains only to fill the empty cells of the bottom right. Consider the partial Latin square $R$ of order $m+1$ based on the symbols $\{1,2, \ldots, m\} \cup\{0\}$ and defined as follows. The filled cells of $R$ will correspond precisely to the filled cells of the bottom right of $Q_{10}$. Symbol $z \in\{1,2, \ldots, m\}$ is in cell $(i, j)$ of $R$ if and only if $(m+i, m+j, z) \in Q_{10}$, and symbol 0 is in cell $(i, j)$ of $R$ if and only if cell $(m+i, m+j)$ of $Q_{10}$ contains a large symbol. Note that symbol 0 occurs in every row of $R$ that contains a filled cell.

If $B=\emptyset$, then $R$ is a partial Latin square of order $m+1$ with two filled cells (corresponding to the two filled cells $\left(\sigma_{r}, H_{1}, r_{1}\right)$ and $\left(\sigma_{r}, C, \sigma_{r-1}+1\right)$ in $\left.Q_{10}\right)$. Since $m \geq 7$, we can apply Theorem 1.1 to complete $R$ to a Latin square of order $m+1$. Otherwise, $B \neq \emptyset$ and $R$ is a partial Latin square of order $m+1$ with one filled row and at most four other filled cells (corresponding to the filled cells $\left(\sigma_{r}, C, \sigma_{r-1}+1\right)$ and $\left(\sigma_{r}, H_{1}, r_{1}\right)$ and possibly $\left(\gamma^{\prime}, D_{l}, y_{l}\right)$ and $\left(\gamma^{\prime}, H_{3}, r_{3}\right)$ in $\left.Q_{10}\right)$. Thus we can apply Lemma 3.8 to obtain a completion of $R$.

Let $R^{\prime}$ be the partial Latin square obtained from $R$ by placing symbol 0 in cell $(i, j)$ if and only if the appended cells $(m+i, n+1)$ and $(n+1, m+j)$ contain the same symbol. Since $R$ is completable and symbol 0 occurs in every row of $R$ that contains a filled cell, $R^{\prime}$ is completable (by Lemma 3.2 with $s=0$ ). Let $R^{\prime \prime}$ be a completion of $R^{\prime}$. We fill each empty cell $(x, y)$ in the bottom right of $Q_{10}$ with the symbol occurring in cell $(x-m, y-m)$ of $R^{\prime \prime}$. To obtain $L^{\prime}$, we then replace each triple $\left(x^{\prime}, y^{\prime}, 0\right)$ with the triple $\left(x^{\prime}, y^{\prime}, z^{\prime}\right)$ where $z^{\prime}$ is the large symbol occurring in the appended cells $\left(x^{\prime}, n+1\right)$ and $\left(n+1, y^{\prime}\right)$.

In both the case $n$ is even and the case $n$ is odd, we obtain a completion of $P$ from $L^{\prime}$ via the following four steps.

(1) If $B \neq \emptyset$ and row $\gamma$ does not set up all switches, then switch in the $\left(\beta, C_{l}, \gamma^{\prime}, D_{l}, x_{l}, y_{l}\right)$-intercalate and switch in the $\left(\beta, C_{i}, \gamma, D_{i}, x_{i}, y_{i}\right)$-intercalate for each $i \in B \backslash\{l\}$.

(2) If $B \neq \emptyset$ and row $\gamma$ sets up all switches, then switch in the $\left(\beta, C_{i}, \gamma, D_{i}, x_{i}, y_{i}\right)$ - 
intercalate for each $i \in B$.

(3) If $D \neq \emptyset$, then switch in the $\left(\beta, W_{j}, \epsilon_{j}, Z_{j}, s_{j}, t_{j}\right)$-intercalate for each $j \in D$.

(4) Switch in the $\left(m+1,2, \sigma_{r}, C, m+1, \sigma_{r-1}+1\right)$-intercalate.

Case 2: Filled row $\alpha$ is in the top and filled row $\beta$ is in the bottom.

We will now segregate row $\beta$ of $P^{\prime}$, if it is not already segregated. Let $k$ be the number of small symbols in the left of row $\beta$ and let $G$ be the swapping graph of $P^{\prime}$. We let $k^{\prime}=k$ if $n$ is even and $k^{\prime}=k+1$ if $n$ is odd. Then let $C_{1}, C_{2}, \ldots, C_{k}, D_{1}, D_{2}, \ldots, D_{k^{\prime}}$, $x_{1}, x_{2}, \ldots, x_{k}$ and $y_{1}, y_{2}, \ldots, y_{k^{\prime}}$ be as defined in Section 5 (see page 12). Let the partite sets of $G$ be $V_{1}=\left\{x_{1}, x_{2}, \ldots, x_{k}\right\}$ and $V_{2}=\left\{y_{1}, y_{2}, \ldots, y_{k^{\prime}}\right\}$.

Recall from Section 5 that $G=K_{k, k^{\prime}}$, and so $G$ contains a matching that includes $V_{1}$. However, certain choices of this matching give rise to problems for particular values of $\alpha, x_{1}, x_{2}, \ldots, x_{k}$ and $y_{1}, y_{2}, \ldots, y_{k^{\prime}}$. More specifically, symbol $m+1$ is a column-reserved symbol, and the occurrence of symbol $m+1$ in row $m$ shall be involved in undoing the $2^{\text {nd }}$ column-segregation swap made in transforming $P$ into $P^{\prime}$. Thus we do not want the occurrence of symbol $m+1$ in row $m$ to be involved in undoing a $\beta$-segregation swap. To avoid this problem, we must consider which cells in the top will be involved in undoing the $\beta$-segregation swaps we shall make (noting that these swaps are determined by the matching in $G$ that includes $V_{1}$ ).

Recall from Section 5 that all but at most one $\beta$-segregation swap will be undone by switching in an intercalate involving row $\gamma$, for some unreserved row $\gamma$ in the top. We choose row $\gamma$ before choosing our matching in $G$ that includes $V_{1}$, as certain choices of row $\gamma$ combined with certain choices of the matching in $G$ give rise to problems (such as loss of independence of $2^{\text {nd }}$ column-segregation and $\beta$-segregation swaps, and the inability to complete the partial Latin square to a segregated Latin square).

We first treat the special case $k=1$. Let row $\gamma$ be a row in the top such that $\gamma \notin\{\alpha, m\}$ and such that cells $(\gamma, 1)$ and $(\gamma, 2)$ do not contain symbol $x_{1}$. Without loss of generality, let the matching that includes $V_{1}$ be $\left\{x_{1} y_{1}\right\}$.

We move now to the case $k \notin\{0,1\}$. To choose row $\gamma$, we first consider the set $T$ of small symbols occurring in the right of row $\beta$ ( so $T=\{1,2, \ldots, m\} \backslash\left\{x_{1}, x_{2}, \ldots, x_{k}\right\}$ ). We choose row $\gamma$ in the top so that cell $(\gamma, 1)$ or cell $(\gamma, 2)$ contains a symbol from $T$, subject to the restriction that $\gamma \notin\{\alpha, m\}$. We claim that such a row exists. Suppose otherwise. Then the only rows in the top which contain symbols from $T$ in columns 1 or 2 are rows $\alpha$ and $m$. It is easy to see that this implies that $T=\{m-1, m\}$ and that $\alpha=m-1$, giving a contradiction to Condition (13) of Definition 4.2.

There is one case in which additional care needs to be taken in choosing row $\gamma$ in order to avoid a technical problem later in the proof (see page 23). Suppose that $k=m-3$ (and so $|T|=3$ ). Then there is a unique column, $J$ say, with $3 \leq J \leq m$ containing a large symbol in row $\beta$. Column $J$ contains a small symbol, $u$ say, in row $\alpha$ (since row $\alpha$ is segregated). If $u \in T$ we require that we choose $\gamma \neq m$ such that cell $(\gamma, 1)$ or cell $(\gamma, 2)$ contains symbol $u$. (To see that such a row exists, note that the occurrence of symbol $u$ in cell $(\alpha, J)$ precludes the occurrence of symbol $u$ in cells $(\alpha, 1)$ or $(\alpha, 2)$. Thus there is 
at least one row other than row $m$ and row $\alpha$ containing symbol $u$ in column 1 or column 2.)

Thus for all values of $k \geq 1$, row $\gamma$ has the desirable property that we can place symbol $x_{i}$ in cell $\left(\gamma, C_{i}\right)$ for at least $k^{\prime}-1$ values of $i \in\{1,2, \ldots, k\}$. If we can place symbol $x_{i}$ in cell $\left(\gamma, C_{i}\right)$ for all $i \in\{1,2, \ldots, k\}$, then we shall say that row $\gamma$ sets up all switches. If row $\gamma$ does not set up all switches, we shall denote by $x_{l}$ the unique symbol in $\left\{x_{1}, x_{2}, \ldots, x_{k}\right\}$ which cannot be placed in row $\gamma$ of $P^{\prime}$.

We can now choose our matching that includes $V_{1}$. The only situation in which we place a restriction on our matching is the following. Suppose that row $\gamma$ does not set up all switches and that $m+1 \in\left\{y_{1}, y_{2}, \ldots, y_{k^{\prime}}\right\}$. Then we choose a matching in $G$ that includes $V_{1}$ so that $m+1$ is not matched with $x_{l}$. Since $G=K_{k, k^{\prime}}$, such a matching exists.

Without loss of generality, let this matching be $\left\{x_{1} y_{1}, x_{2} y_{2}, \ldots, x_{k} y_{k}\right\}$. For brevity, we introduce the set $B=\{1,2, \ldots, k\}$ with $B=\emptyset$ if $k=0$. Let $S^{\prime}$ be the segregated partial Latin square obtained from $P^{\prime}$ by replacing the triples $\left(\beta, C_{i}, x_{i}\right)$ and $\left(\beta, D_{i}, y_{i}\right)$ with $\left(\beta, C_{i}, y_{i}\right)$ and $\left(\beta, D_{i}, x_{i}\right)$ for each $i \in B$. If $n$ is odd, then the unique large symbol in the right of row $\beta$ of $S^{\prime}$ is symbol $y_{k+1}$ in cell $\left(\beta, D_{k+1}\right)$, and we place symbol $y_{k+1}$ in the appended cell $\left(n+1, D_{k+1}\right)$.

We shall now begin constructing a segregated completion $L^{\prime}$ of $S^{\prime}$. To transform $L^{\prime}$ into a completion of $P$, we shall need to be able to undo (in order) the following swaps:

(1) the $\beta$-segregation swaps (if any) made in transforming $P^{\prime}$ into $S^{\prime}$;

(2) the swap of $m+1$ and $\sigma_{r-1}+1$ made in transforming $P$ into $P^{\prime}$.

To do this, we shall ensure that $L^{\prime}$ contains an intercalate containing symbols $x_{i}$ and $y_{i}$ for each $i \in B$ and an intercalate containing symbols $m+1$ and $\sigma_{r-1}+1$ such that a completion of $P$ is obtained by switching in each of these intercalates.

Applying Lemma 3.1, we complete the bottom left of $S^{\prime}$ if $n$ is even, or the augmented bottom left of $S^{\prime}$ if $n$ is odd, with large symbols. We denote the resulting partial Latin square by $Q_{1}$.

We require our completion of $S^{\prime}$ to contain an intercalate containing symbols $x_{i}$ and $y_{i}$ for each $i \in B$. To ensure this, we now fill certain cells in row $\gamma$ of $Q_{1}$. Specifically, we let

$$
Q_{2}=\left\{\begin{array}{l}
Q_{1} \cup\left\{\left(\gamma, C_{i}, x_{i}\right),\left(\gamma, D_{i}, y_{i}\right): i \in B\right\} \text { if } B \neq \emptyset \text { and row } \gamma \text { sets up all switches; } \\
Q_{1} \cup\left\{\left(\gamma, C_{i}, x_{i}\right),\left(\gamma, D_{i}, y_{i}\right): i \in B \backslash\{l\}\right\} \text { if } B \neq \emptyset \text { and row } \gamma \text { does not set up } \\
\quad \text { all switches; } \\
Q_{1} \text { otherwise. }
\end{array}\right.
$$

We next complete the top left of $Q_{2}$ with small symbols to obtain the partial Latin square $Q_{3}$. If $B=\emptyset$, Lemma 3.1 gives the required completion of the top left. Otherwise, we show that we can fill any empty cells in the left of row $\gamma$ with small symbols. By the inductive assumption, the top left of the resulting partial Latin square can then be completed with small symbols. Suppose that the left of row $\gamma$ of $Q_{2}$ is partially filled. Unless $k=m-3$ and row $\gamma$ sets up all switches, there are at least two empty cells in 
the left of row $\gamma$, and we apply Lemma 3.4 to obtain the required completion of the left of row $\gamma$. Hence we can assume that $k=m-3$ and row $\gamma$ sets up all switches. Thus there is a unique empty cell $(\gamma, J)$ in the left of row $\gamma$ in $Q_{2}$. We claim that the unique small symbol not occurring in row $\gamma$ of $Q_{2}$ is allowable for cell $(\gamma, J)$. Suppose otherwise. Then this unique small symbol occurs in the cell $(\alpha, J)$ and is also an element of the set $T=\{1,2, \ldots, m\} \backslash\left\{x_{1}, x_{2}, \ldots, x_{k}\right\}$. But then the restrictions on our choice of row $\gamma$ (see page 21) guarantee that this small symbol occurs in cell $(\gamma, 1)$ or cell $(\gamma, 2)$, and we have a contradiction. Thus we may complete the left of row $\gamma$ and hence the top left of $Q_{2}$.

Unless $B \neq \emptyset$ and row $\gamma$ does not set up all switches, we let $Q_{4}=Q_{3}$. If $B \neq \emptyset$ and row $\gamma$ does not set up all switches, we must ensure that our completion of $S^{\prime}$ contains an intercalate containing symbols $x_{l}$ and $y_{l}$. Let $\gamma^{\prime}$ be the row in which symbol $x_{l}$ occurs in column $C_{l}$ of $Q_{3}$ and let $Q_{4}=Q_{3} \cup\left\{\left(\gamma^{\prime}, D_{l}, y_{l}\right)\right\}$.

If $B=\emptyset$, we let $Q_{5}=Q_{4}$. Otherwise, we will now complete the right of row $\gamma$ with large symbols to obtain the partial Latin square $Q_{5}$. Note that the right of row $\gamma$ contains at most $m-2$ filled cells. If $n$ is even, we apply Lemma 3.4 to the top right when row $\gamma$ sets up all switches, and apply Lemma 3.5 to the top right when row $\gamma$ does not set up all switches, to obtain the required completion of row $\gamma$. If $n$ is odd and row $\gamma$ sets up all switches, we apply Lemma 3.5 to the augmented top right (noting that the appended cell $\left(n+1, D_{k+1}\right)$ is the unique filled cell in row $n+1$ of the augmented top right). Otherwise, $n$ is odd and row $\gamma$ does not set up all switches. In this case, we fill the empty cell $\left(\gamma, D_{l}\right)$ with one of at least $(m+1)-(m-3)-1=3$ allowable large symbols. Lemma 3.5 can then be used to complete the right of row $\gamma$.

We next ensure that our completion will contain an $\left(m, 2, \sigma_{r}, C, m+1, \sigma_{r-1}+1\right)$ intercalate for some $C$. We choose our column $C$ in the right of $Q_{5}$ such that cell $(m, C)$ is allowable for symbol $m+1$ and cell $\left(\sigma_{r}, C\right)$ is allowable for symbol $\sigma_{r-1}+1$. To verify the existence of such a column, we note that there is precisely one occurrence of symbol $\sigma_{r-1}+1$ in the bottom right of $Q_{6}$ (in row $\beta$ ) and that the right of row $\sigma_{r}$ is empty. There is at most one filled cell in the right of row $m+1$ (namely $\left.\left(m+1, D_{l}, y_{l}\right)\right)$, and the choice of matching in $G$ that includes $V_{1}$ guarantees that $y_{l} \neq m+1$. If $n$ is even, there are at most two occurrences of symbol $m+1$ in the top right (in row $\alpha$ and possibly in row $\gamma$ ), and if $n$ is odd there are at most three occurrences of symbol $m+1$ in the augmented top right (recalling that the appended cell $\left(\beta, D_{k+1}\right)$ contains the large symbol $\left.y_{k+1}\right)$. Thus there are at least $m-4$ choices for $C$. Let $Q_{6}=Q_{5} \cup\left\{(m, C, m+1),\left(\sigma_{r}, C, \sigma_{r-1}+1\right)\right\}$.

We now consider separately the case $n$ is even and the case $n$ is odd.

The case $n$ is even for row $\alpha$ in the top and row $\beta$ in the bottom: The top right of $Q_{6}$ consists of one or two filled rows (row $\alpha$ and possibly row $\gamma$ ) and at most two other filled cells (namely $(m, C, m+1)$ and possibly $\left.\left(\gamma^{\prime}, D_{l}, y_{l}\right)\right)$. Thus we may apply Lemma 3.8 to complete the top right of $Q_{6}$ with large symbols. Let the resulting partial Latin square be $Q_{7}$.

To embed $Q_{7}$ in a completion $L^{\prime}$ of $S^{\prime}$, it remains only to fill the empty cells of the bottom right. The bottom right of $Q_{7}$ consists of one filled row (row $\beta$ ) and one other filled cell (namely $\left(\sigma_{r}, C, \sigma_{r-1}+1\right)$ ). Thus we may apply Lemma 3.8 to complete the bottom right of $Q_{7}$ with small symbols. 
The case $n$ is odd for row $\alpha$ in the top and row $\beta$ in the bottom: This case is similar to the case $n$ is even for row $\alpha$ in the top and row $\beta$ in the bottom, except that here we need to place $m$ distinct large symbols in the bottom right (see page 5 ). To do this, we make use of the $2 m+2$ appended cells $(n+1, m+1),(n+1, m+2), \ldots,(n+1, n)$ and $(m+1, n+1),(m+2, n+1), \ldots,(n, n+1)$.

Recall from Section 2 that in a segregated Latin square of odd order, the bottom right contains $m+1$ distinct large symbols, no two of which occur in the same row or the same column. In particular, our segregated completion of $S^{\prime}$ must satisfy this property. The bottom right of $Q_{6}$ contains the unique large symbol $y_{k+1}$ in the cell $\left(\beta, D_{k+1}\right)$ and this large symbol appears in the appended cell $\left(n+1, D_{k+1}\right)$. To begin the process of placing the remaining $m$ large symbols in the bottom right, consider row $\sigma_{r}$ and let $r_{1}$ be the symbol in the appended cell $\left(\sigma_{r}, n+1\right)$. We choose a column, $H_{1}$ say, which is in the right, distinct from column $D_{k+1}$, and for which cell $\left(\sigma_{r}, H_{1}\right)$ is allowable for symbol $r_{1}$. To verify the existence of such a column, we note that there are at most three occurrences of symbol $r_{1}$ in the top right (in row $\alpha$ and possibly in rows $\gamma$ and $\gamma^{\prime}$, noting that cell $\left(\sigma_{r}, 2\right)$ contains symbol $m+1$ and so $\left.r_{1} \neq m+1\right)$. There is precisely one filled cell in the right of row $\sigma_{r}$ (namely $\left(\sigma_{r}, C, \sigma_{r-1}+1\right)$ ). Thus, given the further restriction that $H_{1} \neq D_{k+1}$, there are at least $m-4$ choices for $H_{1}$. We let $Q_{7}=Q_{6} \cup\left\{\left(\sigma_{r}, H_{1}, r_{1}\right)\right\}$ and we place symbol $r_{1}$ in the appended cell $\left(n+1, H_{1}\right)$.

The augmented top right of $Q_{7}$ is a partial Latin square of order $m+1$ containing at most two filled rows and at most four other filled cells (namely $\left(n+1, D_{k+1}, y_{k+1}\right)$, $\left(n+1, H_{1}, r_{1}\right),(m, C, m+1)$ and possibly $\left.\left(\gamma^{\prime}, D_{l}, y_{l}\right)\right)$. Thus we may apply Lemma 3.8 to complete the augmented top right of $Q_{7}$ with large symbols. Let the resulting partial Latin square be $Q_{8}$.

To embed $Q_{8}$ in a completion $L^{\prime}$ of $S^{\prime}$, it remains only to fill the empty cells in the bottom right. Consider the partial Latin square $R$ of order $m+1$ based on the symbols $\{1,2, \ldots, m\} \cup\{0\}$ and defined as follows. The filled cells of $R$ will correspond precisely to the filled cells of the bottom right of $Q_{8}$. Symbol $z \in\{1,2, \ldots, m\}$ is in cell $(i, j)$ of $R$ if and only if $(m+i, m+j, z) \in Q_{8}$, and symbol 0 is in cell $(i, j)$ of $R$ if and only if cell $(m+i, m+j)$ of $Q_{8}$ contains a large symbol. Note that symbol 0 occurs in every row of $R$ that contains a filled cell.

Thus $R$ is a partial Latin square of order $m+1$ with one filled row and two other filled cells (corresponding to the two filled cells $\left(\sigma_{r}, C, \sigma_{r-1}+1\right)$ and $\left(\sigma_{r}, H_{1}, r_{1}\right)$ in $\left.Q_{8}\right)$. Applying Lemma 3.8, we complete $R$ to a Latin square of order $m+1$ based on the symbols $\{0,1,2, \ldots, m\}$.

Let $R^{\prime}$ be the partial Latin square obtained from $R$ by placing symbol 0 in cell $(i, j)$ if and only if the appended cells $(m+i, n+1)$ and $(n+1, m+j)$ contain the same symbol. Since $R$ is completable and symbol 0 occurs in every row of $R$ that contains a filled cell, $R^{\prime}$ is completable (by Lemma 3.2 with $s=0$ ). Let $R^{\prime \prime}$ be a completion of $R^{\prime}$. We fill each empty cell $(x, y)$ in the bottom right of $Q_{8}$ with the symbol occurring in cell $(x-m, y-m)$ of $R^{\prime \prime}$. To obtain $L^{\prime}$, we then replace each triple $\left(x^{\prime}, y^{\prime}, 0\right)$ with the triple $\left(x^{\prime}, y^{\prime}, z^{\prime}\right)$ where $z^{\prime}$ is the large symbol occurring in the appended cells $\left(x^{\prime}, n+1\right)$ and $\left(n+1, y^{\prime}\right)$. 
In both the case $n$ is even and the case $n$ is odd, we obtain a completion of $P$ from $L^{\prime}$ via the following three steps.

(1) If $B \neq \emptyset$ and row $\gamma$ does not set up all switches, then switch in the $\left(\beta, C_{l}, \gamma^{\prime}, D_{l}, x_{l}, y_{l}\right)$-intercalate and switch in the $\left(\beta, C_{i}, \gamma, D_{i}, x_{i}, y_{i}\right)$-intercalate for each $i \in B \backslash\{l\}$.

(2) If $B \neq \emptyset$ and row $\gamma$ sets up all switches, then switch in the $\left(\beta, C_{i}, \gamma, D_{i}, x_{i}, y_{i}\right)$ intercalate for each $i \in B$.

(3) Switch in the $\left(m, 2, \sigma_{r}, C, m+1, \sigma_{r-1}+1\right)$-intercalate.

We can now prove our main result.

Proof of Theorem 1.5. Let $n$ be the order of $P$ and let $m=\left\lfloor\frac{n}{2}\right\rfloor$. The condition that $P$ is not isotopic to either of the two partial Latin squares given in the theorem is clearly necessary for completion of $P$. A computer search has shown that every other partial Latin square of order at most 13 and with two filled rows and two filled columns is completable (the code used in this search can be found in [3]).

For a proof by induction, assume that $n \geq 14$ and that the theorem holds for any partial Latin square of order at most $n-1$. Since $n \geq 14$, we have $m \geq 7$ and so every partial Latin square in $\mathcal{Q}_{m} \cup \mathcal{Q}_{m+1}$ has a completion. By Lemma 4 .3, either $P$ or $P^{T}$ is isotopic to a partial Latin square, $P^{\prime}$ say, with $P^{\prime} \in \mathcal{P}_{n}$. By Lemma 6.1, $P^{\prime}$ is completable. If $P$ is isotopic to $P^{\prime}$ then we are finished by Lemma 4.1. If $P^{T}$ is isotopic to $P^{\prime}$ then $P^{T}$ is completable by Lemma 4.1, and in this case $P$ has completion $L^{T}$ where $L$ is a completion of $P^{T}$.

Acknowledgment. Research supported by the Australian Research Council.

\section{References}

[1] L.D. Andersen and A.J.W. Hilton, Thank Evans!, Proc. London Math. Soc. Third Series (3), 47 (1983), 507-522.

[2] D. Bryant and C.A. Rodger, On the completion of Latin rectangles to symmetric Latin squares, J. Aust. Math. Soc., 76 (2004), 109-124.

[3] M. Buchanan, Embedding, existence and completion problems for Latin squares, PhD thesis, University of Queensland, 2007.

[4] C.J. Colbourn, The complexity of completing partial Latin squares, Discrete Appl. Math., 8 (1984), 25-30.

[5] T. Evans, Embedding incomplete Latin squares, Amer. Math. Monthly, 67 (1960), 958-961. 
[6] R. Häggkvist, A solution of the Evans conjecture for Latin squares of large size, in Combinatorics (Proc. 5th Hungarian Colloq., Keszthely, 1976) Vol. I, Colloq. Math. Soc. Jnos Bolyai, North-Holland, Amsterdam, 18 (1978), 495-513.

[7] M. Hall, An existence theorem for Latin squares, Bull. Amer. Math. Soc., 51 (1945), $387-388$.

[8] P. Hall, On representatives of subsets, J. London Math. Soc., 10 (1935), 26-30.

[9] C.C. Lindner, On completing Latin rectangles, Canad. Math. Bull., 13 (1970), 65-68.

[10] J. Marica and J. Schönheim, Incomplete diagonals of Latin squares, Canad. Math. Bull., 12 (1969), 235.

[11] H.J. Ryser, A combinatorial theorem with an application to Latin rectangles, Proc. Amer. Math. Soc., 2 (1951), 550-552.

[12] B. Smetaniuk, A new construction of Latin squares. I. A proof of the Evans conjecture, Ars Combin., 11 (1981), 155-172. 